\title{
Outcome of the second Medicines Utilisation Research in Africa Group meeting to promote sustainable and appropriate medicine use in Africa Second MURIA Group Workshop and Symposium, University of Botswana, Gaborone, Botswana $25^{\text {th }}$ to $27^{\text {th }}$ July 2016
}

Amos Massele ${ }^{1}$, Johanita Burger ${ }^{2}$, Francis Kalemeera ${ }^{3}$, Mary Jande ${ }^{4}$, Thatayaone Didimalang ${ }^{5}$, Aubrey Chichonyi Kalungia ${ }^{6}$, Kidwell Matshotyana ${ }^{7}$, Michael Law ${ }^{8}$, Brighid Malone ${ }^{9}$, Olayinka Ogunleye ${ }^{10}$, Margaret Oluka ${ }^{11}$, Bene D Anand Paramadhas ${ }^{12,13}$, Godfrey Mutashambara Rwegerera ${ }^{14}$, Sekesai Zinyowera ${ }^{15}$, *Brian Godman ${ }^{16,17}$

1Department of Clinical Pharmacology, School of Medicine, University of Botswana, Gaborone, Botswana. Email: amos.massele@mopipi.ub.bw

${ }^{2}$ Medicine Usage in South Africa (MUSA), Faculty of Health Sciences, North-West University, Potchefstroom, South Africa. Email: johanita.burger@nwu.ac.za

${ }^{3}$ School of Pharmacy, University of Namibia, Windhoek, Namibia. Email: fkalemeera@unam.na ${ }^{4}$ School of Pharmacy, Catholic University of Health and Allied Science, P.O. Box 1464, Mwanza, Tanzania. Emails: maryjande@gmail.com

${ }^{5}$ Gaborone Private Hospital, Gaborone, Botswana. Email:

Thatayaone.Didimalang@lifehealthcare.co.za

6Department of Pharmacy, University of Zambia, Lusaka, Zambia. Email: chichokalungia@gmail.com ${ }^{7}$ Systems for Improved Access to Pharmaceuticals and Services (SIAPS) Program, Pharmaceuticals and Health Technology Group, Management Sciences for Health Swaziland-Mbabane. Email: kmatshotyana@msh.org 8University of British Columbia, Vancouver, Canada and Rwanda Biomedical Center, Kigali, Rwanda. Email: michael.law@ubc.ca

9Bokamoso Private Hospital, Mmopane, Botswana. Emails: Brighid.Malone@bokamosohospital.org ${ }^{10}$ Clinical Pharmacology Unit, Department of Medicine, Lagos State University Teaching Hospital, Ikeja, Lagos, Nigeria. Email: yinkabode@yahoo.com

${ }^{11}$ Department of Pharmacology and Pharmacognosy, School of Pharmacy, University of Nairobi, Nairobi, Kenya. Email: olukamarga@yahoo.com

${ }^{12}$ Department of Pharmacy, Nyangabgwe Hospital, Francistown, Botswana. Email: anandbene@yahoo.com

${ }^{13}$ University of South Africa, Pretoria, South Africa.

${ }^{14}$ Faculty of Medicine, University of Botswana, Gaborone, Botswana. Email: grwege@yahoo.com ${ }^{15}$ Ministry of Health and Child Welfare, Harare, Zimbabwe. Email: zinyowerasekesai@gmail.com ${ }^{16}$ Department of Laboratory Medicine, Division of Clinical Pharmacology, Karolinska Institutet, Karolinska University Hospital Huddinge, SE-141 86, Stockholm, Sweden. Email:

Brian.Godman@ki.se

${ }^{17}$ Strathclyde Institute of Pharmacy and Biomedical Sciences, University of Strathclyde, Glasgow, UK. Email: Brian.godman@strath.ac.uk

*Author for correspondence

Department of Laboratory Medicine, Division of Clinical Pharmacology, Karolinska Institutet, Karolinska University Hospital Huddinge, SE-141 86, Stockholm, Sweden. Email:

Brian.Godman@ki.se. Telephone: +468 8585 81069; Fax: +46 858581070 and Strathclyde Institute of Pharmacy and Biomedical Sciences, University of Strathclyde, Glasgow, UK.

Email:Brian.godman@strath.ac.uk

(Accepted for publication in Expert Review of Pharmacoeconomics and Outcomes Research - Please keep CONFIDENTIAL)

\section{Abstract}

The second MURIA (Medicines Utilisation Research in Africa) group workshop and symposium again brought researchers together from across Africa to improve their knowledge of drug utilisation (DU) methodologies and exchange ideas to further progress DU research in Africa. This built on extensive activities since the first conference including workshops and multiple publications. Anti-infectives were again the principal theme for the 2016 symposium following the workshops. This included presentations regarding strategies to improve antibiotic utilisation among African countries such as point prevalence studies as well as potential ways to reduce self-purchasing of antibiotics. There were 
also presentations on ARVs including renal function and the impact of policy changes. Concerns with adherence to chronic treatments as well as drug: drug interactions and their implications were also discussed. The deliberations resulted in a number of agreed activities before the next conference in Namibia in 2017.

Key words: Adherence, Antibiotics, Africa, ARVs, drug utilisation, rational use of medicines

\section{Manuscript}

MURIA is a multidisciplinary research group striving to promote sustainable, rational medicine use in Africa (1). Activities include the first MURIA meeting in July 2015, activities to improve antibiotic utilisation in Africa (2) and the second MURIA meeting - 25th to 27th July 2016. This was opened by DVCAA University of Botswana (Professor Mokgwathi), who emphasised the importance of MURIA meetings including enhancing the appropriate use of anti-infectives and the training of young scientists to carry out future research.

Delegates subsequently attended either introductory or advanced workshops. Margaret Oluka and Ilse Truter opened the Introductory Drug Utilisation (DU) workshop. Key issues included definitions and classifications, types of DU research, data sources and examples. This was followed by discussions concerning qualitative research applied to DU studies. Key characteristics include its purpose, primary instruments for data collection, inductive process to gather evidence and the product, i.e. the descriptive data. Finally, Marike Cockeran gave a comprehensive workshop on the introduction to statistical techniques applied to DU research.

The advanced workshop was divided into four sessions, two in the morning and two in the afternoon. Marike Cockeran and Michael Law presented the first workshop on advanced statistics including discussions on multilevel models and interrupted time series analyses. Both included examples. The second morning workshop focused on developing formularies and DTCs. Lars Gustafsson discussed the need and role for DTCs illustrated by the 'Wise List' with high adherence rates, driven by robust principles, physician trust and active dissemination $(3,4)$. Kidwell Matshotyana discussed MSH support for developing DTCs including the development of Standard Treatment Guidelines (STGs) in Swaziland (5). Joseph Fadare presented preliminary results of a survey of DTC activities in Nigeria, suggesting a lack of DTCs in many healthcare facilities and often sub-optimal functioning where these exist. Brighid Malone discussed the development of the Bokamoso antibiotic guideline including restricted antibiotics as well as the Antimicrobial Stewardship (AMS) programme.

Andy Gray discussed ways to strengthen research protocols and proposal writing in the afternoon workshops. The other afternoon workshop centred on national and cross national comparative (CNC) studies. Olayinka Ogunleye discussed the challenges of undertaking studies in Nigeria including unrestricted availability of medicines and a lack of coordinated pharmaceutical policies. However there are positive changes including coordinated research published and presented at MURIA conferences $(6,7)$. Lars Gustafsson discussed the efavirenz (EFV) studies in Uganda, already resulting in publications (8). Strengths included utilization of different expertise, expanding collaborations as well as learning more about EFV among African patients, which is a concern (9). Brian Godman finished by discussing lessons learnt from European CNC studies including health authorities learning from the launch of generics as witnessed by the speed of their response to generic ARBs compared with earlier generics $(10,11)$.

The workshops were followed by presentations on antibiotic research in Botswana. Matshediso Matome discussed planned research on the management of URTIs among private GPs in Botswana as they are a critical point for dispensing antibiotics and antibiotics being the most dispensed medicines. Utlwang Toko discussed the AMS programme in Bokamoso hospital among ICU patients, including daily visits from pharmacists as well as implementation of the hospital antibiotic guidelines. Charles Jolomba discussed the AMS programme in Gaborone Private Hospital including reviewing 7 aspects of care such as appropriate dosing, step down from IV to oral and $<4$ antimicrobial prescribed in a given patient. Total bundle compliance was $77.4 \%$ over a 12 month period. Bene Anand Paramadhas finished by discussing the results of the pilot point prevalence study (PPS) initiated following the February 2016 meeting (1). Key points included a high percentage with HIV and treated with HAART (over $90 \%$ in the District Hospital) as well as concerns with the high prevalence of 2 or more catheters. 
Margaret Oluka subsequently discussed ongoing programmes in Kenya to reduce AMR, including optimising antibiotic use and investing in $R \& D$, educational initiatives, surveillance research and antibiotic DU studies. Hannelie Myer discussed planned programmes among public hospitals in South Africa including a planned PPS study, building on activities in Botswana (2). Romuald Mbwasi discussed ongoing programmes in Tanzania including the development of a National multi-sectoral AMR committee. Draft AMR policies have been developed aiming for completion by the end of September 2016. Finally, Sekesai Zinyowera discussed ongoing activities in Zimbabwe including the findings from a recent KAP study, which showed a high number of antibiotics prescribed per patient, exacerbated by patient expectations and physician fears. There were also fears of AMR among pharmacists although over $40 \%$ admitted dispensing without a prescription, the most common reason being the unaffordability of a physician visit.

General DU research in Tanzania included ascertaining drug prescribing in two hospitals in Mwanza (12). Kidwell Matshotyana compared selected prescribing indicators measured before and after the implementation of STGs in Swaziland. Their implementation had a positive but a small level impact on prescribing patterns including reducing the average number of medicines per encounter, reducing physician encounters ending with an antibiotic, reducing the number of injections prescribed and improving STG availability.

Dan Kibbule started the abstract presentations by discussing policies for cotrimoxazole, amoxicillin and azithromycin (CAA) in Namibia's public sector. There are concerns that existing policies promote their wide use. As a result, calls for the development of more comprehensive antibiotic guidelines that are in tandem with AMR patterns. Aubrey Kalungia discussed the prevalence of non-prescription sales and dispensing of antibiotics in community pharmacies in Zambia despite laws forbidding this (13). Dispensing was more prevalent among pharmacy technicians than pharmacists. Sajini Souda presented findings on infant cotrimoxazole (CTX) prophylaxis and found no statistically significant difference in diarrheal disease, pneumonia or death comparing infants with resistant commensal organisms versus sensitive commensal organism. Consequently, concerns that CTX prophylaxis may outweigh the benefits. Francis Kalemerra discussed the effect of changing from first- to second- line antiretroviral therapy on renal function based on his recent paper (14). Michael Law finished this session discussing the impact on ARV use after Rwanda changed the threshold for treatment from CD4 $<350$ to $<500$. This change transiently increased treatment initiation; however, it did not have a major impact on overall treatment rates.

Mwangana Mubita subsequently described the effect of different renal function assessment methods on tenofovir's safety reports. There was a significant lack of agreement between the Cockcroft-Gault (C-G) method and the Chronic Kidney Disease-Epidemiology method, with the C-G method potentially reporting more cases of TDF-associated renal impairment. Consequently, this method should be re-considered. Margaret Oluka discussed ADRs to ARVs. Nevirapine based regimens still the most common for ADRs, with women still bearing the brunt of HIV and ADRs in Kenya similar to other African countries (9). Overall, there is a successful National pharmacovigilance (PV) system in Kenya but the quality of spontaneous reports could be improved. Antionette Terblanché discussed baseline data for the development and evaluation of a structured PV system in Sebokeng Hospital, South Africa. The majority (96.2\%) of professionals felt it necessary to report ADRs, however only a minority did. The most common reason was not knowing how, where and when to report.

Consequently, training for professionals should be improved and reporting forms made easily accessible. Adetutu Ajemigbitse discussed the outcome of an intervention to reduce prescribing errors among tertiary hospitals in Nigeria. Pharmacist-led interventions produced some success. However, the sum effect was not statistically significant. Bene Anand Paramadhas discussed initiatives on medication safety at Nyangabgwe hospital in Botswana. The Pharmacy department chose an Annual Performance Objective of reducing the incidence of actual medication errors to $<1$ per 100,000 items dispensed helped by an independent double check system. Recent programmes appreciably reduced the number of actual errors reaching the patient to $1.85 \%$ of potential errors. Bayobuya Phulu finished Day 2 by discussing ways to promote RUM through active DTCs in Namibia including DTC training, regular ward visits as well as prescriber training on STGs.

Day 3 started with a tribute to Matthias Adorka, MURIA initiator (1), from the Pharmacy Team at the University of Namibia following his untimely death. Mary Jande subsequently discussed adherence rates to antihypertensive among patients attending clinics in Tanzania, which were low compared to 
the required level of $\geq 80 \%$. Educational initiatives are needed to address concerns. Martie Lubbe subsequently discussed medicine possession ratios (MPRm) as proxy for adherence to antiepileptic drugs (AEDs). Further research is needed to determine the factors influencing epileptic patients' low prescription refill adherence rates. Johanita Burger discussed methods to detect potentially serious drug-drug interactions using the Matanović/Vlahović-Palčevski drug-drug interaction protocol as no established protocols for African patients. Johanita concluded this tool might be a useful initial screening tool to prevent the most common potentially serious drug-drug interactions. Ilse Truter discussed vaccination coverage in underprivileged Grade-R school children in Nelson Mandela Bay Health District. Coverage was lower than the average coverage for the Eastern Cape, with a trend linking stunting to incomplete vaccination schedules. Godfrey Rwegerera discussed anti-diabetic medication adherence rates in a tertiary referral centre in Botswana. Overall, adherence rates were suboptimal, mirroring other studies. Only HIV positivity was significantly associated with better medication adherence. Finally, Olayinka Ogunleye presented the results of the African Pharmacology Science Gateway (APSG)/ MURIA survey regarding African Researchers' needs to guide discussions on next steps. Key pertinent areas included continued training in DU and statistics, prescription databases, establishment of PV centres, greater collaboration/ networks and continued funding for conferences. These themes were taken forward and discussed as part of proposed activities for 2016/ 207. All abstracts and workshop presentations can be found on the MURIA website as well as membership forms (http://muria.nmmu.ac.za/).

The feedback from the meeting was positive with attendees typically finding workshop presenters knowledgeable in their areas (4.47 out of 5), the content relevant to their needs and could be applied to their workplace (4.57). Similarly for the symposium at 4.43 and 4.49. The main criticism was not enough time to debate key issues (3.87). We look forward to the third MURIA Group meeting in Namibia in July 2017.

\section{Acknowledgements and financial disclosure}

We would like to thank University of Botswana for the use of their excellent facilities, the administration team at UB for greatly facilitating all aspects of the workshop as well as IUPHAR, Botswana Public Officers' Medical Aid Scheme (BPOMAS) and the Ministry of Health in Botswana for their sponsorship. This meeting was also in part supported by a VR-Link grant from Swedish Research Council (VR-Link 2013-6710) to Amos Y Massele and Lars L Gustafsson.

There are no conflicts of interest from any author.

\section{References}

1. Massele A, Godman B, Adorka M, Fadare J, Gray A, Lubbe M, et al. Initiative to progress research on medicine utilization in Africa: formation of the Medicines Utilization Research in Africa group. Expert review of pharmacoeconomics \& outcomes research. 2015;15(4):607-10.

2. Massele A, Tiroyakgosi C, Matome M, Desta A, Muller A, Paramadhas BA, et al. Research activities to improve the utilization of antibiotics in Africa. Expert review of pharmacoeconomics \& outcomes research. 2016.

3. Gustafsson LL, Wettermark B, Godman B, Andersen-Karlsson E, Bergman U, Hasselstrom J, et al. The 'wise list'- a comprehensive concept to select, communicate and achieve adherence to recommendations of essential drugs in ambulatory care in Stockholm. Basic \& clinical pharmacology \& toxicology. 2011;108(4):224-33.

4. Bjorkhem-Bergman L, Andersen-Karlsson E, Laing R, Diogene E, Melien O, Jirlow M, et al. Interface management of pharmacotherapy. Joint hospital and primary care drug recommendations. European journal of clinical pharmacology. 2013;69 Suppl 1:73-8.

5. Ministry of Health and WHO. Standard Treament Guidelines and Essential Medicines List of Common Medicial Conditions in the Kingdom of Swaziland. Available at URL:

http://apps.who.int/medicinedocs/documents/s22119en/s22119en.pdf. 
6. Fadare JO, Adeoti AO, Desalu OO, Enwere OO, Makusidi AM, Ogunleye O, et al. The prescribing of generic medicines in Nigeria: knowledge, perceptions and attitudes of physicians. Expert review of pharmacoeconomics \& outcomes research. 2015:1-12.

7. Akunne OO, Godman B, Adedapo AD, Truter I, Fadare J. Statin prescribing among hypertensive patients in southwest Nigeria: findings and implications for the future. Journal of comparative effectiveness research. 2016;5(3):281-8.

8. Mukonzo JK, Owen JS, Ogwal-Okeng J, Kuteesa RB, Nanzigu S, Sewankambo N, et al. Pharmacogenetic-based efavirenz dose modification: suggestions for an African population and the different CYP2B6 genotypes. PloS one. 2014;9(1):e86919.

9. Gaida R, Truter I, Grobler C, Kotze T, Godman B. A review of trials investigating efavirenzinduced neuropsychiatric side effects and the implications. Expert review of anti-infective therapy. 2016;14(4):377-88.

10. Moon JC, Godman B, Petzold M, Alvarez-Madrazo S, Bennett K, Bishop I, et al. Different initiatives across Europe to enhance losartan utilization post generics: impact and implications. Frontiers in pharmacology. 2014;5:219.

11. Godman B, Shrank W, Andersen M, Berg C, Bishop I, Burkhardt T, et al. Comparing policies to enhance prescribing efficiency in Europe through increasing generic utilization: changes seen and global implications. Expert review of pharmacoeconomics \& outcomes research. 2010;10(6):707-22. 12. Jande M, Kongola G, Mwangi JW. Drug Prescribing Pattern in Two Hospitals in Mwanza, Northwest Tanzania. East and Central African Journal of Pharmaceutical Sciences. 2012;15:63-8.

13. Kalungia AC, Burger J, Godman B, Costa JO, Simuwelu C. Non-prescription sale and dispensing of antibiotics in community pharmacies in Zambia. Expert review of anti-infective therapy. 2016.

14. Kalemeera F, Mbango C, Mubita M, Naikaku E, Gaida R, Godman B. Effect of changing from first- to second-line antiretroviral therapy on renal function: a retrospective study based on data from a single health facility in Namibia. Expert review of anti-infective therapy. 2016;14(8):777-83. 